\title{
UNIVERSITYOF
}

FORWARD

THINKING

WESTMINSTER用

WestminsterResearch

http://www.westminster.ac.uk/westminsterresearch

\section{Alias-free Discrete-time FIR System Realisation Using Hybrid Stratified Sampling}

Tarczynski, A.

This is a copy of the author's accepted version of a paper subsequently published in the proceedings of 2020 Event-Based Control, Communication and Signal Processing. Krakow, Poland, 23 - 25 Sep 2020, IEEE.

The final published version will be available online at:

https://doi.org/10.1109/EBCCSP51266.2020.9291367

(C) 2020 IEEE . Personal use of this material is permitted. Permission from IEEE must be obtained for all other uses, in any current or future media, including reprinting/republishing this material for advertising or promotional purposes, creating new collective works, for resale or redistribution to servers or lists, or reuse of any copyrighted component of this work in other works.

The WestminsterResearch online digital archive at the University of Westminster aims to make the research output of the University available to a wider audience. Copyright and Moral Rights remain with the authors and/or copyright owners. 


\title{
Alias-free Discrete-time FIR System Realisation Using Hybrid Stratified Sampling
}

\author{
Andrzej Tarczynski \\ School of Computer Science and \\ Engineering \\ University of Westminster \\ London, UK \\ https://orcid.org/0000-0002-7931-6133
}

\begin{abstract}
This paper proposes a method for system realisation, where the realised system is described by a continuous-time, finite-duration impulse response. The proposed discrete-time implementation deploys Digital Aliasfree Signal Processing. It means that despite the use of digital signal processing, the produced results do not suffer from aliasing. However, owing to the use of random sampling, the approach relies on constructing a suitable estimator of the system output. This paper shows that the proposed estimator is unbiased. It is also consistent, i.e. its variance goes to zero when the density of signal samples increasing. It is proven that under moderately restrictive assumptions, the estimator goes to zero proportionally to the fifth power of the average distance between the samples.
\end{abstract}

Keywords-system realisation, alias free sampling, digital alias free signal processing, random sampling, finite impulse response

\section{INTRODUCTION}

It is a well-known fact that the approaches relying on the use of deterministic sampling in digital processing of continuous-time signals suffer from aliasing. In general, this phenomenon can be explained in a trivial manner. For any deterministic sampling pattern, uniform or not, there exist an infinite number of continuous-time signals that take identical values at the sampling instants. If we consider signal sampling as a mathematical process of mapping the space of continuous-time signals to a space of discrete-time ones, aliasing is equivalent to stating that this mapping is not oneto-one. A classical approach to eliminating aliasing is to suitably restrict the class of the processed continuous-time signals and select a matching sampling scheme so that the mapping of the restricted class to the space of discrete-time signals is one-to-one. A textbook solution is to use uniform sampling, where the sampling frequency exceeds the Nyquist rate, which is twice the highest frequency present in the signal. While this approach is guaranteed to work, it may have significant disadvantages affecting its usefulness. First, unless the processed signal is baseband, this approach is likely to result in the use of an excessive sampling rate. Second, all signals are observed in practice only inside a finite-duration window and therefore are not bandlimited. We can say that the above-mentioned, precious one-to-one mapping truly exists only if an infinite number of signal samples is collected. A popular way of eliminating aliasing while sampling the nonbaseband signals at rates below the Nyquist, is to use the Landau rate [1]. The Landau rate is the sampling rate equal to twofold total bandwidth of the signal. Unlike the classical approach, sampling at the Landau rate may involve the use of suitable selected nonuniform sampling. Historically, the first attempt to go this way was to deal with bandpass signals [2]. The support of such signals in the frequency domain is $1_{\left[f_{0}, f_{0}+B\right]}(f)$, where $1_{X}(\cdot)$ is the indicator function defined by
$1_{X}(z)=\left\{\begin{array}{ll}1 & \text { if } z \in X \\ 0 & \text { if } z \notin X\end{array}\right.$. If $f_{0}=p B$, where $p$ is an integer, then such a signal can be sampled uniformly at the Landau rate. Otherwise, aliasing can be avoided by faster sampling rates or using a suitable nonuniform sampling scheme. A more complex task of avoiding aliasing when processing multiband signals was effectively solved with the use periodic nonuniform sampling (PNS), often implemented as multi coset sampling [3]-[4]. The problem with processing signals whose spectral support has such a complex structure is that the positions and widths of individual bands should be known. If this knowledge is not in place, a simple approach is to overestimate spectral support so that it covers the actual one. However, if the bandwidth of the estimated spectral support, is much bigger than the actual signal bandwidth then the resulting sampling rate scheme will be excessive. The solution to this problem comes in a few flavours. Each imposes a significant computational burden when processing the collected data.

Instead of requiring the accurate knowledge of the signal spectral support, these approaches generally rely on two assumptions / pieces of knowledge. The first one is an interval in frequency domain that contains all the spectral components of the processed signal. The second one is the total bandwidth of the signal. The positions and widths of individual spectral components are not needed, although individual methods may impose some restrictions in this aspect. After the signal is sampled, some optimisation algorithm is used to first identify the actual spectral support of the signal and then process the signal. Examples of such approaches involve spectrum- blind / universal PNS [5]-[7] and multi-rate sampling [8]-[9], which are extensions of techniques originally developed for processing multi-bound signals with known spectral support. More generally, all these techniques are referred to as compressive sampling. They involve a variety of approaches widely described in the research literature e.g. [10]-[12]. Digital Alias-free Signal Processing (DASP) approaches the problem of alleviating aliasing from a different angle [13][14]. First of all, it uses alias-free sampling. As we already mentioned, deterministic sampling inherently is susceptible to aliasing. Hence, alias free sampling utilises entirely or partially random sampling. To clarify how this change helps eliminating aliasing, we note that when a continuous-time signal is sampled at random time instants then its discrete-time counterpart is random. If the stochastic properties of such a discrete-time signal are different, whenever the continuoustime originals are different, then the sampling process is aliasfree. To illustrate this concept consider taking only one sample of some signal $v(\cdot)$. Let the probability density function (PDF) of the random time instant be $f_{\tau}(\tau)=\frac{1}{\sqrt{2 \pi}} \exp \left(-\frac{\tau^{2}}{2}\right)$. The random discrete time signal is described by $v_{d}(t ; \hat{\tau})=$ 
$\frac{v(t)}{f_{\tau}(t)} \delta(t-\hat{\tau})$, where $\hat{\tau}$ is the random sampling instant. Let's calculate the expected value of $v_{d}(t ; \hat{\tau}): \mathrm{E}\left\{v_{d}(t ; \hat{\tau})\right\}=$ $\int_{-\infty}^{\infty} f_{\tau}(\tau) v_{d}(t ; \tau) d \tau=f_{\tau}(t) \frac{v(t)}{f_{\tau}(t)}=x(t)$. Since the expected values of discrete-time signals differ from each other as long as the continuous-time originals are different, the above sampling scheme is alias free. We can use the signal sample to construct unbiased estimators of the Fourier transform of $v(\cdot): V_{d}(\omega ; \hat{\tau})=\frac{v(\hat{\tau})}{f_{\tau}(\hat{\tau})} e^{-j \omega \hat{\tau}}$, or the output of the system with impulse response $g(\cdot)$ and input $v(\cdot): y_{d}(t ; \hat{\tau})=\frac{v(\hat{\tau})}{f_{\tau}(\hat{\tau})} g(t-$ $\hat{\tau})$. In fact,

$$
\mathrm{E}\left\{V_{d}(\omega ; \hat{\tau})\right\}=\int_{-\infty}^{\infty} f_{\tau}(\tau) \frac{v(\tau)}{f_{\tau}(\tau)} e^{-j \omega \tau} d \tau=\int_{-\infty}^{\infty} v(\tau) e^{-j \omega \tau} d \tau
$$

and

$$
\begin{aligned}
\mathrm{E}\left\{y_{d}(t ; \hat{\tau})\right\}=\int_{-\infty}^{\infty} f_{\tau}(\tau) \frac{v(\tau)}{f_{\tau}(\tau)} g(t-\tau) d \tau \\
=\int_{-\infty}^{\infty} v(\tau) g(t-\tau) d \tau=y(t)
\end{aligned}
$$

Of course, eliminating of aliasing does not solve all the problems of digital signal processing. The problem that we face now, is that the discrete-time signals to be processed are not fully known. Normally, we access only a single realisation of the random process. Therefore, the focus of DASP, is to select a sampling scheme and construct accurate estimators of the objects (such as Fourier or some other transform or output of a system) that are the aim of signal processing. A popular way of measuring the accuracy is to use the mean squared error, which in the case of unbiased estimators is identical with their variance. For example, in the case of the abovementioned estimators of the Fourier transform and output of the system, the variances are

$$
\begin{aligned}
\sigma_{V_{d}(\omega ; \hat{\tau})}^{2}=\mathrm{E}\left\{\left|V_{d}(\omega ; \hat{\tau})\right|^{2}\right\}-|V(\omega)|^{2} \\
=\int_{-\infty}^{\infty} \frac{v^{2}(\tau)}{f_{\tau}(\tau)} d \tau-|V(\omega)|^{2}
\end{aligned}
$$

and

$$
\begin{aligned}
\sigma_{y_{d}(t ; \hat{\tau})}^{2}=\mathrm{E}\left\{y_{d}^{2}(t ; \hat{\tau})\right\}-y^{2}(t) \\
=\int_{-\infty}^{\infty} \frac{v^{2}(\tau) g^{2}(t-\tau)}{f_{\tau}(\tau)} d \tau-y^{2}(t)
\end{aligned}
$$

In this particular case, both variances could be large and even infinite if the integrals do not have finite values. Therefore, the above example should only be considered as an illustration of how alias-free sampling can be constructed rather than a practical solution to signal processing problems.

Alias free sampling can be traced back to early 1960s when Shapiro and Silverman published their paper [15] on alias-free sampling of random noise. In this paper, they proposed an approach were the power spectrum of a random signal could be determined in an arbitrary range of frequencies while using random sampling at a preselected rate. Their approach was further refined in [16]-[18]. The concept of DASP was systematically explored by Bilinskis and his colleagues. A good summary of their work can be found in two monographs [13]-[14].

The DASP approach was used to solve a variety of problems. Fourier transform estimation of signals observed in a finite window was considered in [19]-[23]. This series of papers shows a gradual improvement in the accuracy of estimators. In this case the accuracy is measured as a function of the number of collected signal samples $N$. The method proposed in [19] used a total random sampling. It was shown that the variance of the estimator goes to zero at the rate $O\left(N^{-1}\right)$. This rate was applicable to both pointwise and uniform (i.e. frequency-independent) convergence. In [20] and [21], by changing the sampling scheme and signal processing algorithms, the pointwise convergence rate as increased to $O\left(N^{-3}\right)$ and $O\left(N^{-5}\right)$ respectively, whereas the uniform convergence rate stayed at $O\left(N^{-1}\right)$. Then the approach proposed in [22] increased the rate of uniform convergence to $O\left(N^{-5}\right)$, and [23] proposed a framework, where with the use of $K$ th-order hybrid stratified sampling, both the pointwise and uniform convergence rates are $O\left(N^{-(2 K+3)}\right)$. The applications of DASP include signal processing for NMR applications [24], spectrum sensing for cognitive radio applications [25]-[27] and digital control [28]. So far, there is limited amount of research devoted to system realisation using DASP. Some preliminary results are shown in [14] and [29]. This paper is one of the initial attempts in this direction. Specifically, we address the issue on using DASP for realisation of linear time-invariant continuous-time systems with finite impulse response. This problem is on itself unique, since traditional realisations of continuous time systems have infinite impulse response, whereas digital realisations can be FIR but suffer from aliasing. The paper is organised as follows. In section II we introduce the problem tackled in this paper, propose a sampling scheme and signal processing algorithm. The properties of the approach are explored in section III. Specifically, we show and prove the necessary and sufficient condition under which our solution is causal - an important factor if the proposed realisation is to operate in real time. We demonstrate that the proposed estimator of the system output is unbiased and show that under very weak assumptions its variance converges to zero at the rate inverse-proportional to the density of samples. However, if the input signal has continuous second derivative, and the impulse response of the system is continuous then this convergence rate increases to the fifth power of the inverse density of samples. In section IV we rewrite the formulas describing the system implementation to make them more suited to practical implementation. To give the reader a feel about the computational workload of the proposed approach at various stages, we split the calculations into two parts: those that have to be performed at the design stage, and then those to be carried our in the real time. Some numerical illustrations of the proposed approach are included in section $\mathrm{V}$. The final discussion is in section VI.

\section{PROBLEM FORMULATION}

This paper is devoted to a DASP realisation of continuoustime, LTIV systems described by

$$
y(t)=\int_{t-T_{H}}^{t-T_{D}} v(\tau) g(t-\tau) d \tau,
$$


where $v(\cdot)$ and $y(\cdot)$ denote the system input and output signal respectively, and the system impulse response $g(\cdot)$ supported by the interval $\left[T_{D}, T_{H}\right)$ is square-integrable, i.e.

$$
G=\int_{T_{D}}^{T_{H}} g^{2}(\tau) d \tau \leq \infty
$$

Below, we describe the proposed sampling scheme and the signal processing algorithm for estimating $y(t)$.

\section{A. Hybrid Sampling Scheme}

The signal $v(\cdot)$ is sampled using hybrid stratified sampling. To this end, the time axis is divided into strata of length $T$. The strata borders are $\tau_{k}=k T$ and the $k$ th stratum is defined by $\Gamma_{k}=\left[\tau_{k}, \tau_{k+1}\right)$. We denote its centre by

$$
c_{k}=\frac{\tau_{k}+\tau_{k+1}}{2}=k T+0.5 T .
$$

Within each stratum, we select a random time instant $\hat{\tau}_{k}=$ $c_{k}+\hat{r}_{k}$, where $\left\{\hat{r}_{k}\right\}_{k=-\infty}^{\infty}$ is a sequence of IID random variables whose PDF is $\frac{1}{T} 1_{[-0.5 T, 0.5 T)}(\cdot)$. The signal $v(\cdot)$ is sampled at each $\tau_{k}$ and $\hat{\tau}_{k}$. We denote $v_{k}=v\left(\tau_{k}\right)$ and $\hat{v}_{k}=$ $v\left(\hat{\tau}_{k}\right)$. The PDF of $\hat{\tau}_{k}$ is

$$
f_{k}(\tau)=\frac{1}{T} 1_{\Gamma_{k}}(\tau)
$$

\section{B. DASP estimator of the system output}

These samples are used to estimate the values of the output $y(\cdot)$ at arbitrarily selected time instants. Before formulating the algorithm for estimating $y(t)$, we put (1) in a form more suited to our needs here. First, we note that since $g(\cdot)$ has finite support, we can write (1) as $y(t)=\int_{\alpha(t)}^{\beta(t)} v(\tau) g(t-$ $\tau) d \tau$, where $\alpha(\cdot)$ and $\beta(\cdot)$ are arbitrary as long as $\alpha(t) \leq t-$ $T_{H}$ and $\beta(t) \geq t-T_{D}$. Here, we choose these to be as close as possible to each other but aligned with strata borders $t_{k}$. This means that $\alpha(t)=k_{b}(t) T, \beta(t)=k_{f}(t) T, k_{b}(t)$ is the largest integer satisfying $k_{b}(t) T \leq t-T_{H}$ and $k_{f}(t)$ is the smallest integer satisfying $k_{f}(t) T \geq t-T_{D}$. Hence

$$
\begin{aligned}
& k_{b}(t)=\left\lfloor\frac{t-T_{H}}{T}\right\rfloor, \\
& k_{f}(t)=\left\lceil\frac{t-T_{D}}{T}\right\rceil,
\end{aligned}
$$

where $[\cdot]$ and $\lceil\cdot T$ are the floor and ceiling function respectively. This allows rewriting (1) as $y(t)=$ $\sum_{k=k_{b}(t)}^{k_{f}(t)-1} \int_{\Gamma_{k}} v(\tau) g(t-\tau) d \tau$. Let's denote

$$
I_{k}(t)=\int_{\Gamma_{k}} v(\tau) g(t-\tau) d \tau
$$

Hence

$$
y(t)=\sum_{k=k_{b}(t)}^{k_{f}(t)-1} I_{k}(t) .
$$

The estimator $\hat{y}(\cdot)$ is constructed as

$$
\hat{y}(t)=\sum_{k=k_{b}(t)}^{k_{f}(t)-1} \hat{I}_{k}(t)
$$

where $\hat{I}_{k}(\cdot)$ are the estimators of $I_{k}(\cdot)$. Let $v_{L I N}(\cdot)$ be a piecewise linear signal defined by

$$
\text { if } t \in \Gamma_{k} \text { then } v_{L I N}(\tau)=a_{k} \times\left(\tau-c_{k}\right)+b_{k},
$$

where $a_{k}=\frac{v_{k+1}-v_{k}}{T}$ and $b_{k}=\frac{v_{k+1}+v_{k}}{2}$. Clearly, as soon as the samples $v_{k}$ and $v_{k+1}$ are known, the signal $v_{L I N}(\cdot)$ can be calculated for any $\tau \in \Gamma_{k}$. We also define $v_{N L}(\cdot)$ :

$$
v_{N L}(\tau)=v(\tau)-v_{L I N}(\tau)
$$

Unlike $v_{\text {LIN }}(\cdot), v_{N L}(\cdot)$ is only available at the sampling instants where the values of $v(\cdot)$ are known. Lemma 1 below lists selected properties of $v_{L I N}(\cdot)$ and $v_{N L}(\cdot)$.

Lemma 1

The signals $v_{L I N}(\cdot)$ and $v_{N L}(\cdot)$ have the following properties

$$
\begin{gathered}
v_{L I N}\left(\tau_{k}\right)=v_{k} \\
v_{L I N}\left(\tau_{k+1}\right)=v_{k+1} \\
v_{N L}\left(\tau_{k}\right)=v_{N L}\left(\tau_{k+1}\right)=0 \\
v_{N L}\left(\hat{\tau}_{k}\right)=\hat{v}_{k}-v_{L I N}\left(\hat{\tau}_{k}\right)
\end{gathered}
$$

If $t \in \Gamma_{k}$ then

$$
\min \left(v_{k}, v_{k+1}\right) \leq v_{L I N}(t) \leq \max \left(v_{k}, v_{k+1}\right)
$$

If $v(\cdot)$ is bounded, i.e. there exists $B>0$ such that for any $\tau$

then for any $\tau$

$$
|v(\tau)| \leq B
$$

and

$$
\left|v_{L I N}(\tau)\right| \leq B
$$

Proof

$$
\left|v_{N L}(\tau)\right| \leq 2 B
$$

Within stratum $\Gamma_{k}, v_{L I N}(\cdot)$ is a linear interpolation of $v(\cdot)$ between $\left(t_{k}, v_{k}\right)$ and $\left(t_{k+1}, v_{k+1}\right)$. This implies (12) - (14), (16) and, with the use of (17), it also proves (18). Then (15) is a direct consequence of (11). Finally, (19) is a simple conclusion from (11), (17) and (18).

It follows from (7), (11) and (10) that

$$
\begin{aligned}
I_{k}(t)=\int_{\Gamma_{k}}\left(a_{k} \times\right. & \left.\left(\tau-c_{k}\right)+b_{k}+v_{N L}(\tau)\right) g(t-\tau) d \tau \\
& =a_{k} \int_{\Gamma_{k}}\left(\tau-c_{k}\right) g(t-\tau) d \tau \\
& +b_{k} \int_{\Gamma_{k}} g(t-\tau) d \tau \\
& +\int_{\Gamma_{k}} v_{N L}(\tau) g(t-\tau) d \tau .
\end{aligned}
$$

Let

$$
\begin{aligned}
\Phi_{k}(t)=\int_{\Gamma_{k}}\left(\tau-c_{k}\right) g(t-\tau) d \tau \\
\quad=\int_{-0.5 T}^{0.5 T} \tau g\left(t-c_{k}-\tau\right) d \tau
\end{aligned}
$$

and

$$
\Psi_{k}(t)=\int_{\Gamma_{k}} g(t-\tau) d \tau=\int_{-0.5 T}^{0.5 T} g\left(t-c_{k}-\tau\right) d \tau,
$$

both of which can be calculated before any signal samples are collected. Then

$$
I_{k}(t)=a_{k} \Phi_{k}(t)+b_{k} \Psi_{k}(t)+\int_{\Gamma_{k}} v_{N L}(\tau) g(t-\tau) d \tau
$$


The integral in (22) is estimated by $\int_{\Gamma_{k}} v_{N L}(\tau) g(t-\tau) d \tau \approx$ $T v_{N L}\left(\hat{\tau}_{k}\right) g\left(t-\hat{\tau}_{k}\right)$. Hence

$$
\hat{I}_{k}(t)=a_{k} \Phi_{k}(t)+b_{k} \Psi_{k}(t)+T v_{N L}\left(\hat{\tau}_{k}\right) g\left(t-\hat{\tau}_{k}\right)
$$

and by (9)

$$
\begin{aligned}
\hat{y}(t)=\sum_{k=k_{b}(t)}^{k_{f}(t)-1} a_{k} \Phi_{k}(t)+b_{k} \Psi_{k}(t) \\
+T v_{N L}\left(\hat{\tau}_{k}\right) g\left(t-\hat{\tau}_{k}\right) .
\end{aligned}
$$

In (24) we add up $N(t)=k_{f}(t)-k_{b}(t)$ components. By (5) and (6), $N(t)=\left\lceil\frac{t-T_{D}}{T}\right\rceil-\left\lceil\frac{t-T_{H}}{T}\right\rfloor$. Let $\delta_{D}=\left\lceil\frac{t-T_{D}}{T}\right\rceil-\frac{t-T_{D}}{T}$ and $\delta_{H}=\frac{t-T_{H}}{T}-\left\lfloor\frac{t-T_{H}}{T}\right\rfloor$. Clearly $\delta_{D}, \delta_{H} \in[0,1)$. We note that $N(t)=\delta_{D}+\frac{t-T_{D}}{T}+\delta_{H}-\frac{t-T_{H}}{T}$, hence

$$
N(t)=\frac{T_{H}-T_{D}}{T}+\delta_{N}
$$

where $\delta_{N}=\delta_{D}+\delta_{H} \in[0,2)$. Even if the length of the stratum $T$ is fixed the number of components in (24) may differ for different time instants $t$. But $N(t)$ is related to the ratio between the length of the impulse response $T_{H}-T_{D}$ and $T$. In particular, when $T \rightarrow 0$, the inverse of $N(t)$ converges to zero at the rate $T$ :

$$
N^{-1}(t)=O(T)
$$

In this paper we explore the properties of the estimators (23) and (24). We demonstrate that they are unbiased and consistent when $T \rightarrow 0$. Also, we explore the rate at which they converge to the target values. Specifically, we show that if $g(\cdot)$ and the second derivative of $v(\cdot)$ are continuous then the variance of $\hat{y}(t)$ is $O\left(T^{5}\right)$.

\section{PROPERTIES OF THE DASP ESTIMATOR OF THE SYSTEMS} OUTPUT

\section{A. Causalty}

A system is causal iff the value of its output never depends on the future values of the input. For example, for the system described by (1) the output $y(t)$ depends on $v(\tau)$, where $\tau \in$ $\left[t-T_{H}, t-T_{D}\right]$. Hence, as long as $T_{D} \geq 0$ this system is causal. Theorem 1 below states when the DASP implementation (24) describes a causal system.

\section{Theorem 1}

The system (24) is causal if $T \leq T_{D}$

Proof

The samples of the input signal needed for calculating $\hat{y}(t)$ are taken at $\left\{\tau_{k}\right\}_{k=k_{b}(t)}^{k_{f}(t)}$ and $\left\{\hat{\tau}_{k}\right\}_{k=k_{b}(t)}^{k_{f}(t)-1}$. The latest sample used in this calculation is taken at $\tau_{k_{f}(t)}=k_{f}(t) T$. The system (24) is causal iff for any $t: k_{f}(t) T \leq t$. By (6), this is equivalent to

$$
\left\lceil\frac{t-T_{D}}{T}\right\rceil \leq \frac{t}{T}
$$

Let $t-T_{D}=\left(n_{t}+\gamma_{t}\right) T$ where $n_{t}$ is an integer and $\gamma_{t} \in$ $[0,1)$. We substitute this in (27) to get

$$
\left\lceil n_{t}+\gamma_{t}\right\rceil \leq n_{t}+\gamma_{t}+\frac{T_{D}}{T}
$$

We note that for any $T_{D}, T$ and $\gamma \in[0,1)$ we can select a time instant $t=(n+\gamma) T+T_{D}$, where $n$ is an arbitrary integer, resulting in $\gamma_{t}=\gamma$. Therefore, (28) must be satisfied for any $\gamma_{t} \in[0,1)$. When $\gamma_{t}=0$, (28) implies $0 \leq T_{D}$.
Hence nonnegative $T_{D}$ is a necessary condition for system causality. If $0<\gamma_{t}<1$ then (28) yields $1 \leq \gamma_{t}+\frac{T_{D}}{T}$, which is satisfied for any $\gamma_{t} \in(0,1)$ iff $\frac{T_{D}}{T} \geq 1$, which proves the theorem.

In the reminder of this section, we focus our attention on the acuracy of the proposed estimator. To this end, we explore the size of its bias and variance. Let the errror of estimating $I_{k}(t)$ be denoted by

$$
\widehat{\Delta}_{k}(t)=\hat{I}_{k}(t)-I_{k}(t) .
$$

By (22) and (23): $\widehat{\Delta}_{k}(t)=T v_{N L}\left(\hat{\tau}_{k}\right) g\left(t-\hat{\tau}_{k}\right)-$ $\int_{\Gamma_{k}} v_{N L}(\tau) g(t-\tau) d \tau$. To simplify notation, for a given output time instant $t$ we define $g_{t}(\tau)=g(t-\tau)$. Therefore

$$
\widehat{\Delta}_{k}(t)=T v_{N L}\left(\hat{\tau}_{k}\right) g_{t}\left(\hat{\tau}_{k}\right)-\int_{\Gamma_{k}} v_{N L}(\tau) g_{t}(\tau) d \tau
$$

\section{B. Lack of bias}

\section{Theorem 2}

For any $t, \hat{I}_{k}(t)$ is an unbiased estimator of $I_{k}(t)$

Proof

We have to prove that $\mathrm{E}\left\{\hat{I}_{k}(t)\right\}=I_{k}(t)$, which is equivalent to $\mathrm{E}\left\{\widehat{\Delta}_{k}(t)\right\}=0$. According to (30)

$$
\mathrm{E}\left\{\widehat{\Delta}_{k}(t)\right\}=T \mathrm{E}\left\{v_{N L}\left(\hat{\tau}_{k}\right) g_{t}\left(\hat{\tau}_{k}\right)\right\}-\int_{\Gamma_{k}} v_{N L}(\tau) g_{t}(\tau) d \tau
$$

Since $\hat{\tau}_{k}$ is a random number whose PDF is given by (4), we get $\quad \mathrm{E}\left\{v_{N L}\left(\hat{\tau}_{k}\right) g_{t}\left(\hat{\tau}_{k}\right)\right\}=\int_{-\infty}^{\infty} f_{k}(\tau) v_{N L}(\tau) g_{t}(\tau) d \tau=$ $\frac{1}{T} \int_{\Gamma_{k}} v_{N L}(\tau) g_{t}(\tau) d \tau$. By substituting this result back into (31) we conclude $\mathrm{E}\left\{\widehat{\Delta}_{k}(t)\right\}=0$.

Theorem 3

For any $t, \hat{y}(t)$ is an unbiased estimator of $y(t)$

Proof

The proof of this Theorem is a simple consequence of (8), (9) and Theorem 2.

\section{Variance of the estimator}

Lack of bias of the proposed estimator is evidence that the proposed sampling scheme and signal processing algorithm are alias-free. This result is valid regardless of how densely the sampling instants are distributed, what the frequencydomain characteristics of the processed signal are or what the shape of the impulse response is. However, as mentioned before, lack of bias does not necessarily mean that that the signal processing results are accurate. In the subsequent analyses we explore the accuracy of the proposed estimator as measured by its variance. Specifically, we relate the size of the variance to the length of the stratum $T$. 
We calculate $\sigma_{\hat{I}_{k}(t)}^{2}$ and $\sigma_{\hat{y}(t)}^{2}$. By (29)-(30) and Theorem 2: $\sigma_{\hat{I}_{k}(t)}^{2}=\mathrm{E}\left\{T^{2} v_{N L}^{2}\left(\hat{\tau}_{k}\right) g_{t}^{2}\left(\hat{\tau}_{k}\right)\right\}-\left[\int_{\Gamma_{k}} v_{N L}(\tau) g_{t}(\tau) d \tau\right]^{2}$.

Since $\mathrm{E}\left\{v_{N L}^{2}\left(\hat{\tau}_{k}\right) g_{t}^{2}\left(\hat{\tau}_{k}\right)\right\}=\frac{1}{T} \int_{\Gamma_{k}} v_{N L}^{2}(\tau) g_{t}^{2}(\tau) d \tau$, we get

$\sigma_{\hat{I}_{k}(t)}^{2}=T \int_{\Gamma_{k}} v_{N L}^{2}(\tau) g_{t}^{2}(\tau) d \tau-\left[\int_{\Gamma_{k}} v_{N L}(\tau) g_{t}(\tau) d \tau\right]^{2}$

For any $t, \hat{I}_{k}(t)$ is a sequence of independent random variables. Therefore, by (9)

$$
\sigma_{\hat{y}(t)}^{2}=\sum_{k=k_{b}(t)}^{k_{f}(t)-1} \sigma_{\hat{I}_{k}(t)}^{2}
$$

Consequently

$$
\begin{aligned}
\sigma_{\hat{y}(t)}^{2}=T & \sum_{k=k_{b}(t)} \int_{\Gamma_{k}} v_{N L}^{2}(\tau) g_{t}^{2}(\tau) d \tau \\
& -\sum_{k=k_{b}(t)}\left[\int_{\Gamma_{k}} v_{N L}(\tau) g_{t}(\tau) d \tau\right]^{2}
\end{aligned}
$$

Theorem 4

If the input signal $v(\cdot)$ is bounded as specified by (17) then $\sigma_{\hat{y}(t)}^{2} \leq 4 T B^{2} G$

Proof

It follows from (34) and (19) that $\sigma_{\hat{y}(t)}^{2} \leq$ $T \sum_{k=k_{b}(t)}^{k_{f}(t)-1} \int_{\Gamma_{k}} v_{N L}^{2}(\tau) g_{t}^{2}(\tau) d \tau \leq$

$4 T B^{2} \sum_{k=k_{b}(t)}^{k_{f}(t)-1} \int_{\Gamma_{k}} g_{t}^{2}(\tau) d \tau=4 T B^{2} \int_{k_{b}(t) T}^{k_{f}(t) T} g_{t}^{2}(\tau) d \tau=$ $4 T B^{2} \int_{t-k_{f}(t) T}^{t-k_{b}(t) T} g^{2}(\tau) d \tau$. Then (5) and (6) yield $t-$ $k_{b}(t) T=t-\left\lfloor\frac{t-T_{H}}{T}\right\rfloor T \geq t-\frac{t-T_{H}}{T} T=T_{H}$,

and $\quad t-k_{f}(t) T=t-\left\lceil\frac{t-T_{D}}{T}\right\rceil T \leq t-\frac{t-T_{D}}{T} T=T_{D} . \quad$ The support of $g^{2}(\cdot)$ is the same as that of $g(\cdot)$, i.e. $\left[T_{D}, T_{H}\right)$. Therefore, by (2) we get $\sigma_{\hat{y}(t)}^{2} \leq 4 T B^{2} \int_{T_{D}}^{T_{H}} g^{2}(\tau) d \tau \leq$ $4 T B^{2} G$.

Theorem 4 implies that when $T \rightarrow 0$ then $\sigma_{\hat{y}(t)}^{2}$ converges to zero, and the convergence rate is at least $O(T)$. In fact, this rate could be much faster. Here, we demonstrate that if the second derivative of $v(\cdot), \ddot{v}(\cdot)$, and the impulse response $g(\cdot)$ are continuous then the rate of convergence of $\sigma_{\hat{y}(t)}^{2}$ is $O\left(T^{5}\right)$. When $\tau \in \Gamma_{k}$ we represent $g_{t}(\cdot)$ and $v(\cdot)$ in the neighbourhood of $c_{k}$ by $g_{t}(\tau)=g_{t}\left(c_{k}\right)+o\left(\left|\tau-c_{k}\right|^{0}\right)$ and $v(\tau)=v\left(c_{k}\right)+\dot{v}\left(c_{k}\right)\left(\tau-c_{k}\right)+\ddot{v}\left(c_{k}\right) \frac{\left(\tau-c_{k}\right)^{2}}{2}+o((\tau-$ $\left.\left.c_{k}\right)^{2}\right)$. Since $\left|\tau-c_{k}\right| \leq \frac{T}{2}$ we can put these relations as

$$
\begin{gathered}
g_{t}(\tau)=g_{t}\left(c_{k}\right)+o\left(T^{0}\right) \\
v(\tau)=v\left(c_{k}\right)+\dot{v}\left(c_{k}\right)\left(\tau-c_{k}\right)+\ddot{v}\left(c_{k}\right) \frac{\left(\tau-c_{k}\right)^{2}}{2}+ \\
o\left(T^{2}\right)
\end{gathered}
$$

By substituting in turn $t_{k}$ and $t_{k+1}$ for $t$ in (36) we get

$$
\begin{gathered}
v_{k}=v\left(c_{k}\right)-\dot{v}\left(c_{k}\right) \frac{T}{2}+\ddot{v}\left(c_{k}\right) \frac{T^{2}}{8}+o\left(T^{2}\right) \\
v_{k+1}=v\left(c_{k}\right)+\dot{v}\left(c_{k}\right) \frac{T}{2}+\ddot{v}\left(c_{k}\right) \frac{T^{2}}{8}+o\left(T^{2}\right)
\end{gathered}
$$

Therefore, $v_{L I N}(\cdot)$ defined by (10) becomes

$$
\begin{aligned}
v_{L I N}(\tau)=v\left(c_{k}\right) & +\dot{v}\left(c_{k}\right)\left(\tau-c_{k}\right)+\ddot{v}\left(c_{k}\right) \frac{T^{2}}{8} \\
& +o\left(T^{2}\right) .
\end{aligned}
$$

By (11), (36) and (39) we get $v_{N L}(\tau)=\ddot{v}\left(c_{k}\right)\left[\frac{\left(\tau-c_{k}\right)^{2}}{2}-\right.$ $\left.\frac{T^{2}}{8}\right]+o\left(T^{2}\right)=\frac{\ddot{v}\left(c_{k}\right)}{2}\left(\tau-c_{k}-\frac{T}{2}\right)\left(\tau-c_{k}+\frac{T}{2}\right)+o\left(T^{2}\right)$. Hence

$$
v_{N L}(\tau)=\frac{1}{2} \ddot{v}\left(c_{k}\right)\left(\tau-\tau_{k+1}\right)\left(\tau-\tau_{k}\right)+o\left(T^{2}\right)
$$

We note that the dominant part of (40) is $O\left(T^{2}\right)$. Therefore, by squaring both sides of (40) we get

$$
v_{N L}^{2}(\tau)=\frac{1}{4} \ddot{v}^{2}\left(c_{k}\right)\left(\tau-t_{k+1}\right)^{2}\left(\tau-t_{k}\right)^{2}+o\left(T^{4}\right)
$$

where the dominant part is $o\left(T^{4}\right)$. Similarly, by squaring both sides of (35) we have

$$
g_{t}^{2}(\tau)=g_{t}^{2}\left(c_{k}\right)+o\left(T^{0}\right)
$$

Now we are ready to calculate $\sigma_{\hat{I}_{k}(t)}^{2}$ as expressed by (32). Let's start with the first component of (32). We use (41) and (42) to get $C_{1}=T \int_{\Gamma_{k}} v_{N L}^{2}(\tau) g_{t}^{2}(\tau) d \tau=T \int_{\Gamma_{k}}\left[\frac{1}{4} \ddot{v}^{2}\left(c_{k}\right)(\tau-\right.$ $\left.\left.t_{k+1}\right)^{2}\left(\tau-t_{k}\right)^{2}+o\left(T^{4}\right)\right]\left[g_{t}^{2}\left(c_{k}\right)+o\left(T^{0}\right)\right] d \tau=$ $\frac{1}{4} T \ddot{v}^{2}\left(c_{k}\right) g_{t}^{2}\left(c_{k}\right) \int_{\Gamma_{k}}\left(\tau-t_{k+1}\right)^{2}\left(\tau-t_{k}\right)^{2} d \tau+o\left(T^{6}\right)$. Since $\int_{\Gamma_{k}}\left(\tau-t_{k+1}\right)^{2}\left(\tau-t_{k}\right)^{2} d \tau=\frac{T^{5}}{30}$, we have

$$
C_{1}=\frac{1}{120} T^{6} \ddot{v}^{2}\left(c_{k}\right) g_{t}^{2}\left(c_{k}\right)+o\left(T^{6}\right) .
$$

Similarly, the second term of (32) is $-C_{2}^{2}$ where $C_{2}=$ $\int_{\Gamma_{k}} v_{N L}(\tau) g_{t}(\tau) d \tau=\int_{\Gamma_{k}}\left[\frac{1}{2} \ddot{v}\left(c_{k}\right)\left(\tau-\tau_{k+1}\right)\left(\tau-\tau_{k}\right)+\right.$ $\left.o\left(T^{2}\right)\right]\left[g_{t}\left(c_{k}\right)+o\left(T^{0}\right)\right] d \tau=\frac{1}{2} \ddot{v}\left(c_{k}\right) g_{t}\left(c_{k}\right) \int_{\Gamma_{k}}(\tau-$ $\left.\tau_{k+1}\right)\left(\tau-\tau_{k}\right) d \tau+o\left(T^{3}\right)$. Since $\quad \int_{\Gamma_{k}}\left(\tau-\tau_{k+1}\right)(\tau-$ $\left.\tau_{k}\right) d \tau=-\frac{T^{3}}{6}$ we get $C_{2}=-\frac{1}{12} T^{3} \ddot{v}\left(c_{k}\right) g_{t}\left(c_{k}\right)+o\left(T^{3}\right)$. The dominant part of $C_{2}$ is $O\left(T^{3}\right)$. Therefore $C_{2}^{2}=$ $\frac{1}{144} T^{6} v^{2}\left(c_{k}\right) g_{t}^{2}\left(c_{k}\right)+o\left(T^{6}\right)$. By substituting $C_{1}$ and $C_{2}^{2}$ in (32) we get $\sigma_{\hat{I}_{k}(t)}^{2}=\frac{1}{120} T^{6} \ddot{v}^{2}\left(c_{k}\right) g_{t}^{2}\left(c_{k}\right)-$ $\frac{1}{144} T^{6} \ddot{v}^{2}\left(c_{k}\right) g_{t}^{2}\left(c_{k}\right)+o\left(T^{6}\right)$, hence

$$
\sigma_{\hat{I}_{k}(t)}^{2}=\frac{1}{720} T^{6} \ddot{v}^{2}\left(c_{k}\right) g_{t}^{2}\left(c_{k}\right)+o\left(T^{6}\right) .
$$

Now we use (33) to calculate $\sigma_{\hat{y}(t)}^{2}=$ $\frac{1}{720} T^{6} \sum_{k=k_{b}(t)}^{k_{f}(t)-1} \ddot{v}^{2}\left(c_{k}\right) g_{t}^{2}\left(c_{k}\right)+\sum_{k=k_{b}(t)}^{k_{f}(t)-1} o\left(T^{6}\right)$. Since in the second summation the number of components is $N(t)$ then by (26) $\sigma_{\hat{y}(t)}^{2}=\frac{1}{720} T^{6} \sum_{k=k_{b}(t)}^{k_{f}(t)-1} \ddot{v}^{2}\left(c_{k}\right) g_{t}^{2}\left(c_{k}\right)+o\left(T^{5}\right)=$ $\frac{1}{720} T^{6} \sum_{k=k_{b}(t)}^{k_{f}(t)-1} \ddot{v}^{2}\left(c_{k}\right) g^{2}\left(t-c_{k}\right)+o\left(T^{5}\right)$. Let's calculate $\sigma^{2}(t)=\lim _{T \rightarrow 0} \frac{\sigma_{\widehat{y}(t)}^{2}}{T^{5}}=\frac{1}{720} \lim _{T \rightarrow 0} \sum_{k=k_{b}(t)}^{k_{f}(t)-1} T \ddot{v}^{2}\left(c_{k}\right) g_{t}^{2}\left(c_{k}\right)+$ $\lim _{T \rightarrow 0} \frac{o\left(T^{5}\right)}{T^{5}}$. We use the Riemann integration to calculate the first component. Its value is $\frac{1}{720} \int_{t-T_{D}}^{t-T_{H}} \ddot{v}^{2}(\tau) g_{t}^{2}(\tau) d \tau$. The second component is clearly 0 . Hence we conclude that

$$
\sigma^{2}(t)=\frac{1}{720} \int_{t-T_{D}}^{t-T_{H}} \ddot{v}^{2}(\tau) g^{2}(t-\tau) d \tau
$$

and that for sufficiently small $T$ 


$$
\sigma_{\hat{y}(t)}^{2} \approx \frac{T^{5}}{720} \int_{t-T_{D}}^{t-T_{H}} \ddot{v}^{2}(\tau) g^{2}(t-\tau) d \tau
$$

\section{THE ALGORITHM FOR SYSTEM REALISATION}

In the previous sections we presented mathematical formulas related to DASP implementation of the filter in the forms that were best suited for analysing its properties. In this section, we rephrase these to clarify how to implement a system in DASP. In our discussions we assume that the system impulse response $g(\cdot), T_{D}, T_{H}$ and the length of the stratum $T$ are already selected. The clue for system implementation is given by (23) and (24). We need to select the time instants $t$, for which the output will be calculated, find out the range of the index $k$ in (24), and calculate $\Phi_{k}(t)$ and $\Psi_{k}(t)$. As we show below, this work can be done off-line. Then, once the suitable signal samples are collected we need to calculate $a_{k}$, $b_{k}, v_{N L}\left(\hat{\tau}_{k}\right)$ and $g\left(t-\hat{\tau}_{k}\right)$ and use these in (24) or an equivalent formula to calculate the output of the system.

The output of the system will be derived for the time instants defined by $t_{n, l}=n T+t_{l}, n=0,1, \ldots, l=1, \ldots, L$ where $0 \leq t_{1}<t_{2}<\cdots<t_{L}<T$. We start with (5) and (6) to calculate $k_{b}(\cdot)$ and $k_{f}(\cdot)$ for the selected output time instants. We note $k_{b}\left(t_{n, l}\right)=\left\lfloor\frac{n T+t_{l}-T_{H}}{T}\right\rfloor=n+\left\lfloor\frac{t_{l}-T_{H}}{T}\right\rfloor$. Hence

Similarly,

$$
k_{b}\left(t_{n, l}\right)=n+k_{b}\left(t_{l}\right)
$$

$$
k_{f}\left(t_{n, l}\right)=n+k_{f}\left(t_{l}\right)
$$

Let $\delta_{k}=k-n$. Hence $\Phi_{k}(\cdot)=\Phi_{n+\delta_{k}}(\cdot)$ and $\Psi_{k}(\cdot)=$ $\Psi_{n+\delta_{k}}(\cdot)$ With this notation we can re-write (9)

$$
\begin{array}{r}
\hat{I}_{k}\left(t_{n, l}\right)=a_{k} \Phi_{n+\delta_{k}}\left(t_{n, l}\right)+b_{n+\delta_{k}} \Psi_{n+\delta_{k}}\left(t_{n, l}\right) \\
+T v_{N L}\left(\hat{\tau}_{k}\right) g\left(t_{n, l}-\hat{\tau}_{k}\right)
\end{array}
$$

We use (20) to calculate $\Phi_{n+\delta_{k}}\left(t_{n, l}\right)=\int_{-0.5 T}^{0.5 T} \tau g\left(n T+t_{l}-\right.$ $\left.c_{n+\delta_{k}}-\tau\right) d \tau$. Since $c_{n+\delta_{k}}=n T+c_{\delta_{k}}$, we get $\Phi_{k}\left(t_{n, l}\right)=$ $\int_{-0.5 T}^{0.5 T} \tau g\left(t_{l}-c_{\delta_{k}}-\tau\right) d \tau$ and

$$
\Phi_{n+\delta_{k}}\left(t_{n, l}\right)=\Phi_{\delta_{k}}\left(t_{l}\right)
$$

Similarly, $\quad \Psi_{n+\delta_{k}}\left(t_{n, l}\right)=\int_{-0.5 T}^{0.5 T} g\left(t_{n, l}-c_{n+\delta_{k}}-\tau\right) d \tau=$ $\int_{-0.5 T}^{0.5 T} g\left(t_{l}-c_{\delta_{k}}-\tau\right) d \tau$ and

$$
\Psi_{n+\delta_{k}}\left(t_{n, l}\right)=\Psi_{\delta_{k}}\left(t_{l}\right)
$$

By substituting (49) and (50) in (48) we get

$$
\begin{aligned}
\hat{I}_{k}\left(t_{n, l}\right)=a_{k} \Phi_{\delta_{k}}\left(t_{l}\right)+b_{k} \Psi_{\delta_{k}}\left(t_{l}\right) & \\
& +T v_{N L}\left(\hat{\tau}_{k}\right) g\left(t_{n, l}-\hat{\tau}_{k}\right)
\end{aligned}
$$

Recalling $\hat{\tau}_{k}=c_{k}+\hat{r}_{k}$ we get from (10) and (11) $v_{N L}\left(\hat{\tau}_{k}\right)=$ $v\left(\hat{\tau}_{k}\right)-v_{L I N}\left(\hat{\tau}_{k}\right)=\hat{v}_{k}-a_{k}\left(\hat{\tau}_{k}-c_{k}\right)-b_{k}=\hat{v}_{k}-a_{k} \hat{r}_{k}-$ $b_{k}$, and $g\left(t_{n, l}-\hat{\tau}_{k}\right)=g\left(n T+t_{l}-c_{k}-\hat{r}_{k}\right)=g(n T+$ $\left.t_{l}-c_{n+\delta_{k}}-\hat{r}_{k}\right)=g\left(n T+t_{l}-n T-c_{\delta_{k}}-\hat{r}_{k}\right)=g\left(t_{l}-\right.$ $\left.c_{\delta_{k}}-\hat{r}_{k}\right)$. We use these expressions to expand (51): $\hat{I}_{k}\left(t_{n, l}\right)=a_{k} \Phi_{\delta_{k}}\left(t_{l}\right)+b_{k} \Psi_{\delta_{k}}\left(t_{l}\right)+T\left(\hat{v}_{k}-a_{k} \hat{r}_{k}-\right.$ $\left.b_{k}\right) g\left(t_{l}-c_{\delta_{k}}-\hat{r}_{k}\right)=\frac{v_{k+1}-v_{k}}{T} \Phi_{\delta_{k}}\left(t_{l}\right)+\frac{v_{k+1}+v_{k}}{2} \Psi_{\delta_{k}}\left(t_{l}\right)+$ $T\left(\hat{v}_{k}-\frac{v_{k+1}-v_{k}}{T} \hat{r}_{k}-\frac{v_{k+1}+v_{k}}{2}\right) g\left(t_{l}-c_{\delta_{k}}-\hat{r}_{k}\right)=$ $v_{k+1}\left[\frac{\Phi_{\delta_{k}}\left(t_{l}\right)}{T}+\frac{\Psi_{\delta_{k}}\left(t_{l}\right)}{2}-\left(\hat{r}_{k}+0.5 T\right) g\left(t_{l}-c_{\delta_{k}}-\hat{r}_{k}\right)\right]+$ $v_{k}\left[-\frac{\Phi_{\delta_{k}}\left(t_{l}\right)}{T}+\frac{\Psi_{\delta_{k}}\left(t_{l}\right)}{2}+\left(\hat{r}_{k}-0.5 T\right) g\left(t_{l}-c_{\delta_{k}}-\hat{r}_{k}\right)\right]+$ $T \hat{v}_{k} g\left(t_{l}-c_{\delta_{k}}-\hat{r}_{k}\right)$. Therefore,

$$
\begin{aligned}
\hat{y}\left(t_{n, l}\right)=\sum_{k=n+k_{b}\left(t_{l}\right)}^{n+k_{f}\left(t_{l}\right)-1}\left(\alpha_{n, k, l}-\hat{\alpha}_{n, k, l}\right) v_{k+1} \\
\quad+\left(\beta_{n, k, l}+\hat{\beta}_{n, k, l}\right) v_{k}+\hat{\gamma}_{n, k, l} \hat{v}_{k}
\end{aligned}
$$

where

$$
\begin{gathered}
\alpha_{n, k, l}=\frac{\Phi_{\delta_{k}}\left(t_{l}\right)}{T}+\frac{\Psi_{\delta_{k}}\left(t_{l}\right)}{2} \\
\hat{\alpha}_{n, k, l}=\left(\hat{r}_{k}+0.5 T\right) g_{k} \\
\beta_{n, k, l}=-\frac{\Phi_{\delta_{k}}\left(t_{l}\right)}{T}+\frac{\Psi_{\delta_{k}}\left(t_{l}\right)}{2} \\
\hat{\beta}_{n, k, l}=\left(\hat{r}_{k}-0.5 T\right) \hat{g}_{k} \\
\hat{\gamma}_{n, k, l}=T \hat{g}_{k} \\
\hat{g}_{k}=g\left(t_{l}-c_{\delta_{k}}-\hat{r}_{k}\right)
\end{gathered}
$$

and $\delta_{k}$ is a shortcut notation for $\delta_{k}=k-n$.

Based on these calculations we propose an algorithm for calculating the output $\hat{y}\left(t_{n, l}\right)$ for any integer $n$ and $l=$ $1, \ldots, L$. The algorithm consists of two parts. The first one is preparatory and can be executed before the system realization is put in work to produce the output. The second part is executed in real time, every time when a new stratum-load of input signal samples is available.

\section{Algorithm - Part 1 executed off-line}

Step 1 . For every $l=1, \ldots, L$

Step 2. Use (5) and (6) to calculate $k_{b}\left(t_{l}\right)$ and $k_{f}\left(t_{l}\right)$

Step 3. For every $\delta_{k}=k_{b}\left(t_{l}\right), \ldots, k_{f}\left(t_{l}\right)-1$

Step 4. Use (20) to calculate

$$
\Phi_{\delta_{k}}\left(t_{l}\right)=\int_{-0.5 T}^{0.5 T} \tau g\left(t_{l}-c_{\delta_{k}}-\tau\right) d \tau
$$

Step 5. Use (21) to calculate

$$
\Psi_{\delta_{k}}\left(t_{l}\right)=\int_{-0.5 T}^{0.5 T} g\left(t_{l}-c_{\delta_{k}}-\tau\right) d \tau
$$

Step 6. Use (53) to calculate $\alpha_{\delta_{k}, l}$

Step 7. Use (55) to calculate $\beta_{\delta_{k}, l}$

\section{Algorithm - Part 2 executed in real time}

Step 1 . For every $l=1, \ldots, L$

Step 2. For every $\delta_{k}=-k_{b}\left(t_{l}\right), \ldots,-k_{f}\left(t_{l}\right)+1$

Step 3. Calculate $g_{l, k}=g\left(t_{l}-(k-n+0.5) T-\hat{r}_{k}\right)$

Step 4. Use (54) to calculate $\hat{\alpha}_{n, k, l}$

Step 5. Use (56) to calculate $\hat{\beta}_{n, k, l}$

Step 6. Use (57) to calculate $\hat{\gamma}_{n, k, l}$

Step 7. Calculate $\hat{I}_{k}\left(t_{n, l}\right)=\left(\alpha_{k-n, l}-\hat{\alpha}_{n, k, l}\right) v_{k+1}+$ $\left(\beta_{k-n, l}-\hat{\beta}_{n, k, l}\right) v_{k}+\hat{\gamma}_{n, k, l} \hat{v}_{k}$

Step 8. Calculate $\hat{y}\left(t_{n, l}\right)=\sum_{k=n+k_{b}\left(t_{l}\right)}^{n+k_{f}\left(t_{l}\right)-1} \hat{I}_{k}\left(t_{n, l}\right)$

\section{NUMERICAL EXAMPLES}

In the examples shown in this section, we numerically explore the properties of the DASP system implementation proposed in this paper. In our experiments we implement a system whose impulse response is a delayed and truncated sinc function: $g(\tau)=\operatorname{sinc}(\tau-4) 1_{[1,7]}$ and the input signal is $v(\tau)=5 \exp \left(-\frac{t^{2}}{4}\right)+0.4 \cos (6 \pi t)$.

In the first example we implement the system twice. In the first attempt the stratum length is $T=0.5$, and in the second $T=0.2$. Fig. 1 shows the output signal of the system. Owing to the use of random sampling, the results of experiments with 
the same implementation of the system differ from case to case. Therefore, each implementation is tested ten times and all ten recorded outputs are shown. Also, the target response of the system, calculated from (1), is superimposed on the plots in this figure to illustrate the size of the error.

(a)

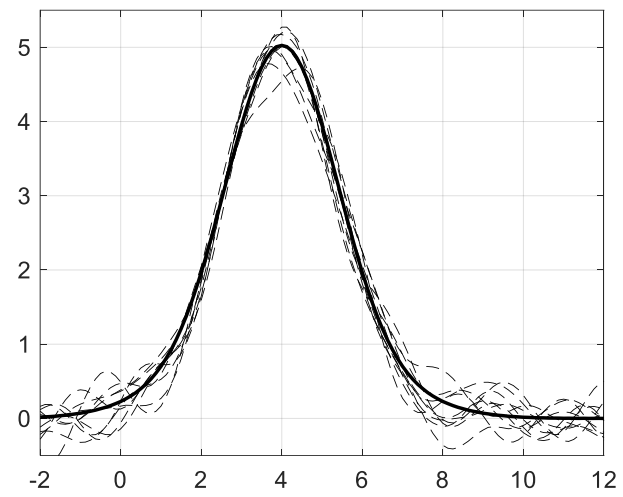

(b)

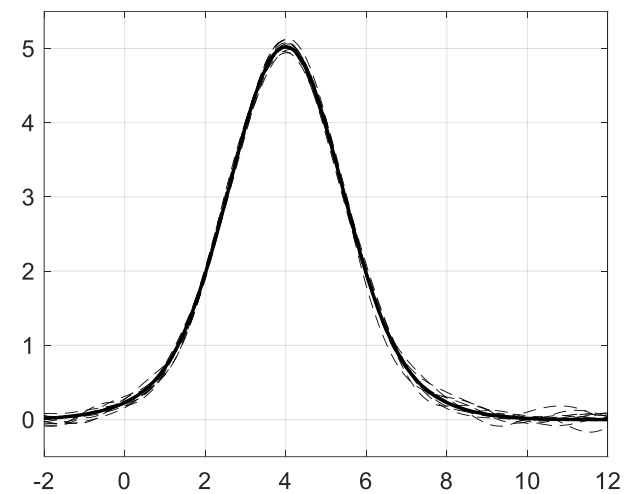

Fig. 1. The response of the system. The target response (thick continuous line) and ten responses of DASP system realization (thin broken lines) for (a) $T=0.5$ and (b) $T=0.2$

The results show that DASP implementation provides a good approximation of the system output in the observed interval and that reducing the length of stratum clearly improves the quality of the results.

In the second experiment we focus our attention on the output signal at $t=4$, and change the length of the stratum from $T=1$ down to $T=10^{-2.5} \approx 0.00316$. Fifty stratum lengths distributed logarithmically are tested. For each length we run 1000 experiments and use these to estimate the variance of the output generated by the DASP system realization. Note that in the previous sections we only derived the asymptotic variance of the estimator. Therefore, it is interesting to see the actual shape of the curve representing the relation between $T$ and $\sigma_{\hat{y}(4)}^{2}$. This is shown in Fig. 2. The results confirm the property of DASP that the increase or decrease of density of the processed-signal samples results in gradual improvement or deterioration of the results. This is different from non-alias free approaches, where occurrence of aliasing can rapidly destroy the quality signal processing. We also note that the slope of curve in Fig. 2 for shorter strata confirms our earlier prediction that the rate by which the variance goes down to zero is $O\left(T^{-5}\right)$.

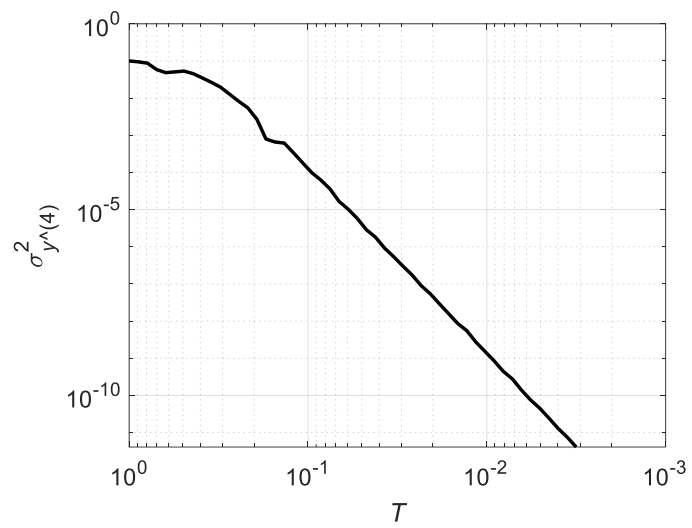

Fig 2. The size of the variance of $\hat{y}(4)$ as function of $T$

\section{SUMMARY AND CONCLUSIONS}

In this paper we proposed a method for DASP realisation of continuous-time, linear, time-invariant systems with finite impulse response. In this case we used an equal-length-strata version of the hybrid stratified sampling and developed signal processing algorithm to produce an unbiased estimator of the system output. We showed the conditions, under which the proposed realisation is causal. We also showed two rates of convergence of the estimate depending on the smoothness of the input signal and the impulse response of the system. While we did not explore any practical constraints on the alias-free features of the proposed approach, we are aware of some of them. The DASP works in its full capacity if the random time instants can take any value within the given range. Of course, many practical solutions will replace this with selecting random sampling instants from a dense uniform grid. This simplification will immediately impose an upper bound on the spectral support of the processed signal $v(\cdot)$, although it does not limit the spectral support of $g(\cdot)$. Second, similarly to what was reported in [19] any jitter in timing of sampling instants also imposes the limit on the bandwidth within which the DASP results are accurate.

There are still questions that need to be answered. For example, for a specific scenario, how to best choose the length of the stratum? The analyses in this paper give plenty of clues but do not provide a simple answer. Next, how to incorporate some knowledge about the signal? e.g. some knowledge of spectral occupancy of the processed signal. While classical DSP relies on accurate knowledge of the bandwidth(s) of the signal, DASP is almost completely indifferent to this information. Each approach can claim some benefits from making its assumptions but also suffers in terms of quality of the results or what it can deliver. In reality we deal with scenarios that are somewhere in between both extremes. The knowledge of processed signals has often "fuzzy logic" form. This partial knowledge should be incorporable into signal processing algorithms to remove strict restrictions imposed by the Nyquist and Landau rates while further improving the quality of results obtainable from DASP.

\section{REFERENCES}

[1] H. J. Landau, "Sampling, Data Transmission, and the Nyquist Rate," Proceedings of the IEEE, vol. 55, pp. 1701-1706, October 1967

[2] R. G. Vaughan, N. L. Scott, and D. R. White, "The theory of bandpass sampling," IEEE Transactions on Signal Processing, vol. 39, pp. 1973 -1984 , September 1991.

[3] R. Venkataramani, and Y. Bresler, "Optimal sub-Nyquist nonuniform sampling and reconstruction for multiband signals," IEEE Transactions on Signal Processing, vol. 49, pp. 2301-2313, October 2001. 
[4] S. C. Scoular, and W. J. Fitzgerald, "Periodic nonuniform sampling of multiband signals," Signal Processing, vol. 28, pp. 195-200, August 1992.

[5] P. Feng, and Y. Bresler, "Spectrum-blind minimum-rate sampling and reconstruction of multiband signals," IEEE International Conference on Acoustics, Speech, and Signal Processing, pp. 1688-1691, May 1996.

[6] D. D. Ariananda, G. Leus, and Z. Tian, "Multi-coset sampling for power spectrum blind sensing," 17th International Conference on Digital Signal Processing (DSP), pp. 1-8, July 2011.

[7] M. E. Domínguez-Jiménez, N. González-Prelcic, G. Vazquez-Vilar, and R. López-Valcarce, "Design of universal multicoset sampling patterns for compressed sensing of multiband sparse signals," IEEE International Conference on Acoustics Speech and Signal Processing, pp. 3337-3340, March 2012.

[8] M. Fleyer, A. Linden, M. Horowitz, and A. Rosenthal, "Multirate Synchronous Sampling of Sparse Multiband Signals," IEEE Transactions on Signal Processing, vol. 58, pp. 1144 - 1156, March 2010.

[9] M. E. Domínguez-Jitnénez, and N. González-Prelcic, "Analysis and design of multirate synchronous sampling schemes for sparse multiband signals,", 20th European Signal Processing Conference, pp. 1184-1188, August 2012.

[10] E. J. Candes, and M. B. Wakin, "An Introduction To Compressive Sampling," IEEE Signal Processing Magazine, vol. 25, pp. 21-30, March 2008

[11] R. G. Baraniuk, V. Cevher, M. F. Duarte, and C. Hegde, "Model-Based Compressive Sensing," IEEE Transactions on Information Theory, vol. 56, pp. 1982-2001, April 2010.

[12] M. Mishali, and Y. C. Eldar, "From Theory to Practice: Sub-Nyquist Sampling of Sparse Wideband Analog Signals," IEEE Journal of Selected Topics in Signal Processing, vol. 4, pp. 375-391, April 2010.

[13] I. Bilinskis and A. K. Mikelson, Randomized Signal Processing. Prentice-Hall, Englewood Cliffs, NJ 1992

[14] I. Bilinskis, Digital Alias-free Signal Processing. John Wiley \& Sons, Hoboken, NJ 2006.

[15] H. S. Shapiro, and R. A. Silverman, "Alias-Free Sampling of Random Noise," Journal of the Society for Industrial and Applied Mathematics, vol. 8, pp. 225-248, June 1960.

[16] E. Masry, "Random sampling and reconstruction of spectra," Information and Control, vol. 19, pp 275-288, November 1970.

[17] F. Beutler, "Alias-free randomly timed sampling of stochastic processes," IEEE Transactions on Information Theory, vol. 16, pp. 147-152, March 1970.
[18] E. Masry, "Alias-free sampling: An alternative conceptualization and its applications," IEEE Transactions on Information Theory, vol. 24, pp. 317-324, May 1978.

[19] A. Tarczynski and N. Allay, "Spectral analysis of randomly sampled signals: suppression of aliasing and sampler jitter", IEEE Trans. Signal Processing, vol. 52, no. 12, pp. 3324-3334, December 2004.

[20] E. Masry, "Random sampling of deterministic signals: statistical analysis of Fourier transforms estimates", IEEE Trans. Signal Processing, vol. 54, pp. 1750-1761, 2006.

[21] E. Masry and A. Vadrevu, "Random sampling estimates of Fourier transforms: antithetical stratified Monte Carlo", IEEE Trans. Signal Processing, vol. 57, pp. 149-204, 2009.

[22] A. Tarczynski and B. I. Ahmad, "Estimation of Fourier Transform Using Alias-Free Hybrid-Stratified Sampling", IEEE Trans. Signal Processing, vol. 64, pp. 3065 - 3076, 2016.

[23] M. Al-Ani, A. Tarczynski and B. I. Ahmad "High-order hybrid stratified sampling: fast uniform-convergence Fourier transform estimation", 52nd Asilomar Conf. Signals, Systems, and Computers, 2018.

[24] K. Kazimierczuk, A. Zawadzka, W. Koźmiński and I. Zhukov, "Random sampling of evolution time space and Fourier transform processing", Journal of Biomolecular NMR, vol. 36, pp. 147-168, 2006.

[25] H. Semlali, N. Boumaaz, A. Soulmani, A. Ghammaz, and J-F. Diouris, "Energy Detection Approach for Spectrum Sensing in Cognitive Radio Systems with the Use of Random Sampling," Wireless Personal Communications, vol. 79, pp. 1053-1061, July 2014.

[26] B. I. Ahmad, and A. Tarczynski, "Wideband spectrum sensing technique based on random sampling on grid: Achieving lower sampling rates," Digital Signal Processing, vol. 21, pp. 466-476, May 2011 .

[27] B. I. Ahmad and A. Tarczynski, "Spectral analysis of stratified sampling: a means to perform efficient multiband spectrum sensing", IEEE Trans. Wireless Communications, vol. 11, pp. 178 - 187, 2012.

[28] M. S. Khan, R. M. Goodall, and R. Dixon, "Design and analysis of nonuniform rate digital controllers," UKACC International Conference on Control 2010, Coventry, 2010, pp. 1-6.

[29] H. Darawsheh, and A. Tarczynski, "FIR Filtering of Discontinuous Signals: A Random-Stratified Sampling Approach," IEEE Int. Conference on Acoustics, Speech, and Signal Processing, May 2020. 